\title{
La enseñanza y aprendizaje de la geometría en secundaria, la perspectiva de los estudiantes
}

\section{The Students' Perspective of Geometry Teaching and Learning in High School}

\author{
Ronny Gamboa Araya ${ }^{l}$ \\ Escuela de Matemática de la Universidad Nacional de Costa Rica \\ Heredia, Costa Rica \\ rgamboa@una.ac.cr \\ Esteban Ballestero Alfaro ${ }^{2}$ \\ Escuela de Ciencias y Letras del Instituto Tecnológico de Costa Rica \\ San Carlos, Costa Rica \\ eballestero@gmail.com
}

Recibido 25 de marzo de 2010 • Aceptado 24 de junio de 2010

\begin{abstract}
Resumen. El propósito de este artículo es presentar los resultados obtenidos con la aplicación de un cuestionario dirigido a estudiantes de secundaria de Costa Rica para conocer su percepción sobre la enseñanza y aprendizaje de la geometría. Los resultados muestran que las clases de geometría en la educación secundaria se han basado en un sistema tradicional de enseñanza, donde docentes presentan la teoría, desarrollan ejemplos y aportan los ejercicios que deben ser resueltos por estudiantes. Estas actividades enfatizan en la aplicación de fórmulas y aspectos memorísticos, lo que trae como consecuencia que procesos de visualización, argumentación y justificación no tengan un papel preponderante en la enseñanza de la disciplina. La geometría se presenta a las estudiantes y los estudiantes como un conjunto de definiciones, fórmulas y teoremas totalmente alejado de su realidad y donde los ejemplos y ejercicios no poseen ninguna relación con su contexto, consecuentemente, la geometría se percibe como poco importante, ya que no es aplicable a la vida cotidiana, cuando la realidad es otra. Además, el grupo estudiantil considera que para tener éxito en geometría hay que saber utilizar la calculadora para realizar cálculos, tener capacidad para memorizar definiciones, fórmulas y teoremas, poseer capacidad para entender los dibujos geométricos y realizar listas de ejercicios para desarrollar la habilidad práctica.
\end{abstract}

Palabras clave. Enseñanza, aprendizaje, geometría, estudiantes.

\footnotetext{
Máster en Ciencias en la especialidad de Matemática Educativa del Centro de Investigación y de Estudios Avanzados del Instituto Politécnico Nacional de México. Licenciado en la Enseñanza de la Matemática de la Universidad Nacional de Costa Rica. Actualmente es el director de la Escuela de Matemática de la Universidad Nacional de Costa Rica.

2 Máster en Ciencias en la especialidad de Matemática Educativa del Centro de Investigación y de Estudios Avanzados del Instituto Politécnico Nacional de México. Bachiller en la Enseñanza de la Matemática asistida por Computadora del Instituto Tecnológico de Costa Rica. Actualmente es profesor de la Escuela de Matemática de la Universidad Nacional de Costa Rica y del Instituto Tecnológico de Costa Rica, sede Santa Clara, San Carlos.
} 
Abstract. The purpose of this article is to present the results obtained from a questionnaire applied to Costa Rican high school students, in order to know their perspectives about geometry teaching and learning. The results show that geometry classes in high school education have been based on a traditional system of teaching, where the teacher presents the theory; he presents examples and exercises that should be solved by students, which emphasize in the application and memorization of formulas. As a consequence, visualization processes, argumentation and justification don't have a preponderant role. Geometry is presented to students like a group of definitions, formulas, and theorems completely far from their reality and, where the examples and exercises don't possess any relationship with their context. As a result, it is considered not important, because it is not applicable to real life situations. Also, the students consider that, to be successful in geometry, it is necessary to know how to use the calculator, to carry out calculations, to have capacity to memorize definitions, formulas and theorems, to possess capacity to understand the geometric drawings and to carry out clever exercises to develop a practical ability.

Key words. Teaching, learning, geometry, students.

\section{Introducción}

La geometría ha sido considerada como uno de los pilares de formación académica y cultural del individuo, dada su aplicación en diversos contextos; su capacidad formadora del razonamiento lógico (Báez e Iglesias, 2007); y su contribución en el desarrollo de habilidades para visualizar, pensar críticamente, intuir, resolver problemas, conjeturar, razonar deductivamente y argumentar de manera lógica en procesos de prueba o demostración (Jones, 2002).

Hernández y Villalba (2001) brindan una visión de la geometría como:

- La ciencia del espacio, vista esta como una herramienta para describir y medir figuras, como base para construir y estudiar modelos del mundo físico y fenómenos del mundo real.

- Un método para las representaciones visuales de conceptos y procesos de otras áreas en matemáticas y en otras ciencias; por ejemplo, gráficas y teoría de gráficas, histogramas, entre otros.

- Un punto de encuentro en una matemática teórica y una matemática como fuente de modelos.

- Una manera de pensar y entender.

- Un ejemplo para la enseñanza del razonamiento deductivo.

- Un modelo para la enseñanza del razonamiento deductivo.

- Una herramienta en aplicaciones, tanto tradicionales como innovadoras, como por ejemplo, gráficas por computadora, procesamiento y manipulación de imágenes, reconocimiento de patrones, robótica, investigación de operaciones.

Además, autores como Castiblanco, Urquina, Camargo y Acosta (2004) señalan que el desarrollo histórico de la geometría ha estado relacionado con actividades humanas, sociales, culturales, científicas y tecnológicas; situación que puede utilizarse para justificar un re-direccionamiento de los procesos de enseñanza hacia el logro de una visión contextualizada de la geometría, la cual, a diferencia de la percepción disjunta que concibe su evolución de forma enajenada de la dinámica social, se oriente a potenciar su aplicabilidad y utilidad en la vida del ser humano, así como a incentivar en los estudiantes y las estudiantes el desarrollo de ciertas habilidades, entre ellas, razonamiento y justificación (National Council of Teachers of Mathematics [NCTM], 2000). 
No obstante, en el sistema de educación formal, en primaria y secundaria, usualmente los contenidos de geometría son presentados al estudiantado como el producto acabado de la actividad matemática. La enseñanza tradicional de esta disciplina se ha enfatizado en la memorización de fórmulas para calcular áreas y volúmenes, así como definiciones geométricas, teoremas y propiedades, apoyadas en construcciones mecanicistas y descontextualizadas.

Incluso autores como Abrate, Delgado y Pochulu (2006) señalan que algunas docentes y algunos docentes priorizan la enseñanza de las matemáticas en otras áreas y van desplazando los contenidos de geometría hacia el final del curso, lo que les implica, en variados casos, la exclusión de estos temas o su atención de manera superficial. La enseñanza de la geometría con este enfoque ha provocado que esta sea considerada como una disciplina difícil y poco útil para la mayoría estudiantil.

Esta situación deja entrever que la enseñanza de la disciplina no está logrando los objetivos deseados, pues la geometría se puede considerar como un instrumento reflexivo que le permite al ser humano resolver problemas de diversa índole y comprender el mundo en cada uno de los escenarios que lo conforman, sea este natural o artificial. Incluso, autores como Almeida (2002) señalan la existencia de algunos objetivos generales que toda persona debería alcanzar durante su formación básica: tener una cultura geométrica con visión histórica e interdisciplinaria, aplicar conocimientos geométricos para modelar, crear o resolver problemas reales, usar los diferentes lenguajes y representaciones, entre otros.

Aunque existen diversas investigaciones sobre la evolución del conocimiento y el aprendizaje, específicamente en el área de geometría, las diferentes situaciones que se presentan en las aulas evidencian la necesidad, por parte de docentes y estudiantes, de promover un aprendizaje efectivo (Goncalves, 2006).

Para realizar ese cambio efectivo que permita un mayor aprendizaje es necesario tener una visión general del contexto actual de la enseñanza y aprendizaje de la geometría, lo cual permitirá tomar acciones tendientes a mejorar y corregir los posibles errores.

Con el objetivo de lograr una visión general de la situación actual de la enseñanza y aprendizaje de la geometría en la educación secundaria costarricense, y con el propósito de conocer su opinión como actores fundamentales del proceso, se aplicó un cuestionario dirigido a estudiantes y docentes.

En el presente artículo se muestran los resultados obtenidos con 233 estudiantes de secundaria, de todos los niveles. Primeramente se presentan algunos referentes teóricos respecto al aprendizaje y enseñanza de la geometría y los procesos de visualización y justificación (fundamentales en el aprendizaje de la disciplina).

Luego se realiza una breve descripción de la metodología utilizada para la aplicación del cuestionario y del proceso empleado para el análisis de los datos. Posteriormente se presenta el análisis de la información recolectada y se finaliza con algunas reflexiones y conclusiones a partir de los resultados obtenidos.

\section{El aprendizaje y enseñanza de la geometría}

De acuerdo con el Costa Rica. Ministerio de Educación Pública (2005), en la enseñanza de la geometría se debe combinar la intuición, experimentación y la lógica. Además, se debe utilizar construcciones para caracterizar las figuras, para que, a partir de estas, el estudiantado formule deducciones lógicas. 
Báez e Iglesias (2007) señalan seis principios didácticos que consideran fundamentales dentro de los procesos de enseñanza y aprendizaje de la geometría:

- $\quad$ Principio globalizador o interdisciplinar: Consiste en un acercamiento conciente a la realidad, donde todos los elementos están estrechamente relacionados entre sí.

- Integración del conocimiento: El conocimiento no está fragmentado, sino que representa un saber integrado, lo que implica también una integración de los objetivos, contenidos, metodología y la evaluación.

- Contextualización del conocimiento: Los conocimientos son adaptados a las necesidades y características de las estudiantes y los estudiantes, a partir del uso de hechos concretos.

- $\quad$ Principio de flexibilidad: La organización y administración del proceso educativo debe ser adaptable a las necesidades del alumnado, sin perder de vista el logro de los objetivos propuestos.

- $\quad$ Aprendizaje por descubrimiento: Todo proceso de enseñanza debe considerar una participación activa del estudiantado, de manera que propicie la investigación, reflexión y búsqueda del conocimiento.

- Innovación de estrategias metodológicas: El grupo docente debe buscar y emplear estrategias metodológicas que incentiven al alumnado hacia la investigación, descubrimiento y construcción del aprendizaje.

Por otra parte, Veloso (1998), (citado por Almeida, 2002), hace también un aporte en esta dirección, señalando que la enseñanza de la geometría en secundaria debe:

- Profundizar y sintetizar los aspectos geométricos en desarrollo, como la comprensión del espacio y de los respectivos modelos geométricos que son dados por las matemáticas; es decir, partir de problemas y situaciones relacionadas con el espacio, como la simetría, la forma y la dimensión.

- Integrar la historia de la geometría en su enseñanza, para permitir al alumnado tener la noción de la existencia de otras geometrías.

- Buscar la conexión de la geometría con otras ramas de las matemáticas, con otras disciplinas como el arte y promover su aplicabilidad en contextos reales.

El NCTM (2000), dentro de su visión de estandarización de la enseñanza de las matemáticas, aporta directrices para orientar la enseñanza de la geometría desde la enseñanza preescolar hasta la secundaria. Esta propuesta gira en torno a cuatro objetivos generales, para los cuales existen objetivos específicos en cada nivel. Los objetivos generales son:

- Analizar las características y propiedades de figuras geométricas de dos y tres dimensiones y desarrollar razonamientos matemáticos sobre relaciones geométricas.

- Localizar y describir relaciones espaciales mediante coordenadas geométricas y otros sistemas de representación.

- $\quad$ Aplicar transformaciones y usar la simetría para analizar situaciones matemáticas.

- Utilizar la visualización, el razonamiento matemático y la modelización geométrica para resolver problemas.

De dicha propuesta se puede observar que los procesos de descripción, comprensión, análisis, construcción, exploración, visualización, argumentación, aplicación, entre otros, deben 
ser implementados en la enseñanza de la geometría mediante el planteamiento de situaciones problema que impliquen, para el alumnado, un nivel cognitivo no limitado al uso de una fórmula o proceso algorítmico.

Sin embargo, y a pesar de que las distintas propuestas señalan la importancia de la enseñanza de la geometría y dan algunas pautas para ello, frecuentemente la enseñanza de esta disciplina se ha limitado a reconocer figuras y dibujarlas en el papel. Las lecciones se han desarrollado de manera abstracta, sin proporcionarles a las estudiantes y a los estudiantes ejemplos reales o contextualizados que les faciliten un mejor entendimiento de los contenidos (Goncalves, 2006). Además, los recursos utilizados para la enseñanza de la geometría son limitados y se circunscriben a los "tradicionales", pues en la mayoría de los casos el proceso de enseñanza está condicionado por los libros de texto, que impactan considerablemente en el qué y cómo enseñar (Abrate et al., 2006).

Aunque las docentes y los docentes, en su mayoría, son conscientes de la importancia de la geometría como una disciplina aplicable a muchos contextos y situaciones reales, el alumnado se encuentra en una disyuntiva cuando estudia la disciplina, pues si bien el profesorado les dice que es importante para su futuro como individuo, el mismo proceso educativo en el que se encuentra inmerso no le permite visualizar esa importancia con suficiente claridad; de manera que el aprendizaje de la geometría carece de sentido y con el tiempo repercute en su estado anímico (Báez e Iglesias, 2007).

Barrantes (2004) señala que en las últimas décadas la enseñanza de la geometría se caracterizaba por:

- Una fuerte tendencia a la memorización de conceptos y propiedades que muchas veces se basan en conceptos previos.

- La resolución automática de problemas en la que se tratan aspectos aritméticos.

- Una exclusión de la intuición, demasiado pronto, como acceso al conocimiento geométrico.

Báez e Iglesias (2007) señalan que, a nivel de educación básica, la enseñanza de las matemáticas presenta dificultades, particularmente la enseñanza y aprendizaje de la geometría, pues algunas veces las docentes y los docentes no desarrollan los contenidos geométricos contemplados en los programas ya sea por desconocimiento de la importancia de la disciplina o por poco dominio de los contenidos geométricos. En aquellos casos en que sí se desarrollan, se hace enfatizando en el uso de fórmulas y cálculo de áreas.

Al respecto, Goncalves (2006) señala que aunque las estudiantes y los estudiantes pueden resolver problemas concretos con bastante habilidad, carecen de estrategias de solución cuando se enfrenten a las mismas situaciones planteadas en otros contextos diferentes, abstractos o más formalizados. Otra situación típica “(...) es la de los estudiantes que tienen que recurrir a memorizar las demostraciones de los teoremas o las formas de resolver los problemas, pues es la única manera de llegar a aprobar los exámenes" (Goncalves, 2006, p. 90).

Si bien la importancia de la enseñanza de la geometría radica en ser la disciplina donde el estudiantado lleva a cabo procesos de razonamiento, la situación que se da en las aulas es distinta; pues uno de los problemas en la enseñanza de la geometría es la dificultad que existe para que las estudiantes y los estudiantes pasen de la descripción de las figuras a un proceso más formal, basado en razonamientos y argumentación (Castiblanco et al., 2004).

¿Cuál es la visión de las estudiantes y los estudiantes que han pasado por los procesos educativos formales, de la primaria y secundaria, sobre su experiencia directa al estudiar geometría? Barrantes y Blanco $(2005,2004)$ señalan algunas concepciones que estudiantes ya graduados poseen acerca de la enseñanza de la geometría: 
- $\quad$ Consideran que la finalidad de la enseñanza de la geometría es adquirir conocimiento, ya sea por cultura general o porque es una parte de las matemáticas y todas son importantes.

- Conciben la geometría escolar como una materia difícil, a la que se dedica poco tiempo.

- Señalan que la geometría es una materia muy teórica, abstracta y complicada de entender, para la que se necesita una mayor capacidad de razonamiento.

- $\quad$ Para el estudiantado la dificultad de la geometría radica, principalmente, en la memorización de fórmulas y saber cuándo aplicarlas.

- Indican que para aprender geometría es necesario la explicación de la profesora o profesor y la práctica, pues si se es capaz de resolver las prácticas se puede verificar si se comprendió el tema en estudio.

- $\quad$ Revelan que la metodología clásica para la enseñanza de la geometría se divide en dos: la parte teórica, caracterizada por definiciones, propiedades, entre otros, y la parte práctica, donde se entienden como sinónimos las palabras problema y ejercicio.

- $\quad$ Apuntan que los contenidos que más se estudian son los relacionados con la geometría plana; en la geometría espacial se profundiza menos.

- Manifiestan que la pizarra y el libro de texto son los recursos más utilizados para la enseñanza de la geometría.

- $\quad$ Destacan que el uso de materiales como figuras de madera u otros son poco frecuentes y cuando se utilizan se hacen construcciones o actividades sin ninguna utilidad posterior.

- Declaran que las actividades geométricas frecuentemente son extraídas del libro de texto y suelen estar relacionadas con el estudio de elementos de las figuras, clasificación y sobre todo de medida; es decir, resolución de problemas "tradicionales".

- Indican que el examen es el elemento más importante de la evaluación.

Lo anterior nos indica que la enseñanza de esta disciplina se ha inscrito en un ambiente aislado del entorno del estudiante, donde los contenidos no representan un conocimiento útil para este y donde el ensayo, el error y la discusión no son aprovechados como un medio para lograr un aprendizaje.

Esta problemática en la enseñanza de la geometría plantea, a todos los involucrados en el proceso de enseñanza y aprendizaje de las matemáticas, un reto para hallar diferentes alternativas de solución, pues se ha desvirtuado la enseñanza de esta disciplina y se han dejado de lado procesos de razonamiento, argumentación y visualización, trascendentales para el aprendizaje de la geometría.

\section{Procesos de visualización y justificación}

El aprendizaje de la geometría implica el desarrollo de habilidades visuales y de argumentación. Más aún, para lograr un aprendizaje significativo es necesario construir una interacción fuerte entre estos dos componentes, de manera que el discurso teórico quede anclado en experiencias perceptivas que ayuden a construir su sentido y, a su vez, las habilidades visuales deben ser guiadas por la teoría, para ganar en precisión y potencia (Castiblanco et al., 2004).

Estos autores, además, mencionan que el aprendizaje de la geometría se centra principalmente en tres aspectos:

a) Los procesos de visualización (que constituyen el soporte de la actividad cognitiva en geometría donde la estudiante y el estudiante "evolucionan" en su percepción de los objetos) y su potencial heurístico en la resolución de problemas. 
b) Los procesos de justificación propios de la actividad geométrica.

c) El papel que poseen las construcciones geométricas en el desarrollo del conocimiento geométrico.

Para que el aprendizaje de la geometría no carezca de sentido, es importante que el grupo docente se preocupe por buscar un equilibrio entre la asociación de habilidades de visualización y argumentación, pues ambas habilidades son fundamentales dentro del proceso formativo del individuo. Es decir, no se trata sólo de enseñar contenidos como una "receta" o por cumplir con lo estipulado en el currículo sino que se pretende que con la enseñanza de la geometría el estudiantado aprenda a pensar lógicamente.

El ser humano, desde su infancia, crea representaciones del mundo físico que le rodea. Estas le generan una necesidad (teórica y práctica) para lograr el entendimiento de ese mundo. El hemisferio derecho del cerebro resulta ser el más beneficiado ante la presencia de estímulos visuales, a diferencia del hemisferio izquierdo, que tiene la responsabilidad de desarrollar las capacidades verbales.

El estudio de la geometría contribuye significativamente al desarrollo de esas necesidades espaciales de visualización; sin embargo, hasta una época histórica reciente, que data a partir de la década de los años 50, es cuando educadores matemáticos se interesaron por el estudio de dicho campo, al vincular la capacidad matemática con la capacidad espacial.

Aunque se ha reconocido la importancia de la capacidad aritmética y el desarrollo de la capacidad de razonamiento, los contenidos geométricos asociados a la capacidad espacial han sido desplazados a un segundo plano en importancia, pues prácticamente desapareció de los planes de estudio durante la época de los años sesenta y setenta, como consecuencia del posicionamiento de las llamadas "Matemáticas modernas", caracterizadas por su formalismo y la algebrización de la geometría.

Torregrosa y Quesada (2007) señalan que, en el estudio de la geometría, se denomina visualización al proceso o acción de transferencia de un dibujo a una imagen mental de un objeto (que no tiene que ser igual para todos) o viceversa. Es decir, un dibujo de un cuadrado (que está sujeto a ciertas definiciones y propiedades) condiciona en cada individuo una imagen mental de este, que va estar asociada a ciertas afirmaciones matemáticas que el mismo individuo le proporciona.

La definición anterior menciona que la visualización es, ante todo, un proceso; esto implica que debe darse de forma paulatina. La persona primeramente se ve expuesta ante un dibujo geométrico estático, pero cuando esta empieza a extraer relaciones o identificar algunas propiedades que le permiten conocer, con mayor profundidad, aquel dibujo, entonces se dice que está visualizando. La figura estática empezó a tener más sentido y mentalmente empieza a construir una imagen más compleja, que va más allá de los trazos externos. La capacidad de ver más allá de lo descriptivo en un dibujo geométrico, identificando propiedades y comprendiendo su interrelación, resume los elementos que pueden derivarse a partir de la visualización.

Clements y Battista (1992), (citado en Castiblanco et al., 2004), consideran que “(...) la visualización integra los procesos por medio de los cuales se obtienen conclusiones a partir de las representaciones de los objetos bi o tridimensionales observadas en construcciones y manipulaciones" (p. 10).

Por ejemplo, Gamboa y Morales (2008), basados en Castiblanco et al., 2004), proponen el siguiente ejemplo en el cual, después de un proceso de visualización a partir de una representación gráfica, se puede realizar un proceso de justificación de la conjetura obtenida. 
Sea el rectángulo $\square A B C D$, donde $\overline{B D}$ es una de sus diagonales (Figura 1).

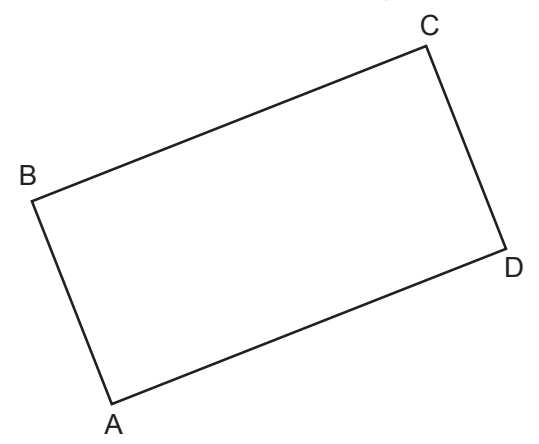

Figura 1. Rectángulo $\square A B C D$

Si sobre el segmento $\overline{B D}$ se construye un punto $I$ y, además, se construyen los puntos $E, F, G, H$ tales que $A-E-B, B-F-C, C-G-D, A-H-D, \overline{B C}\|\overline{E G}, \overline{A B}\| \overline{F H} y \overline{B D} \cap \overline{E G} \cap \overline{F H}=\{I\}$ (Figura 2), ¿qué se puede deducir de las áreas de $\square A E I H$ y $\square I F C G$ ?

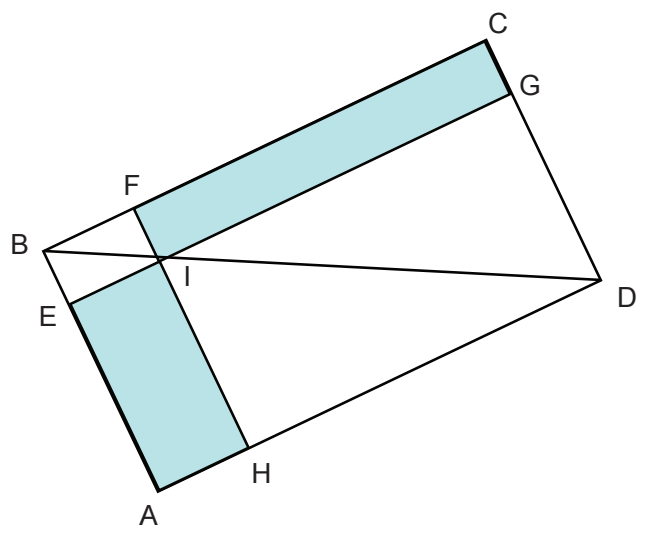

Figura 2. Rectángulo con vértices $A, B, C$ y $D$.

Dada la representación anterior, se puede investigar qué relación tienen las áreas de los cuadriláteros $\square A E I H$ y $\square I F C G$.

Visualmente, se podría dar la siguiente solución.
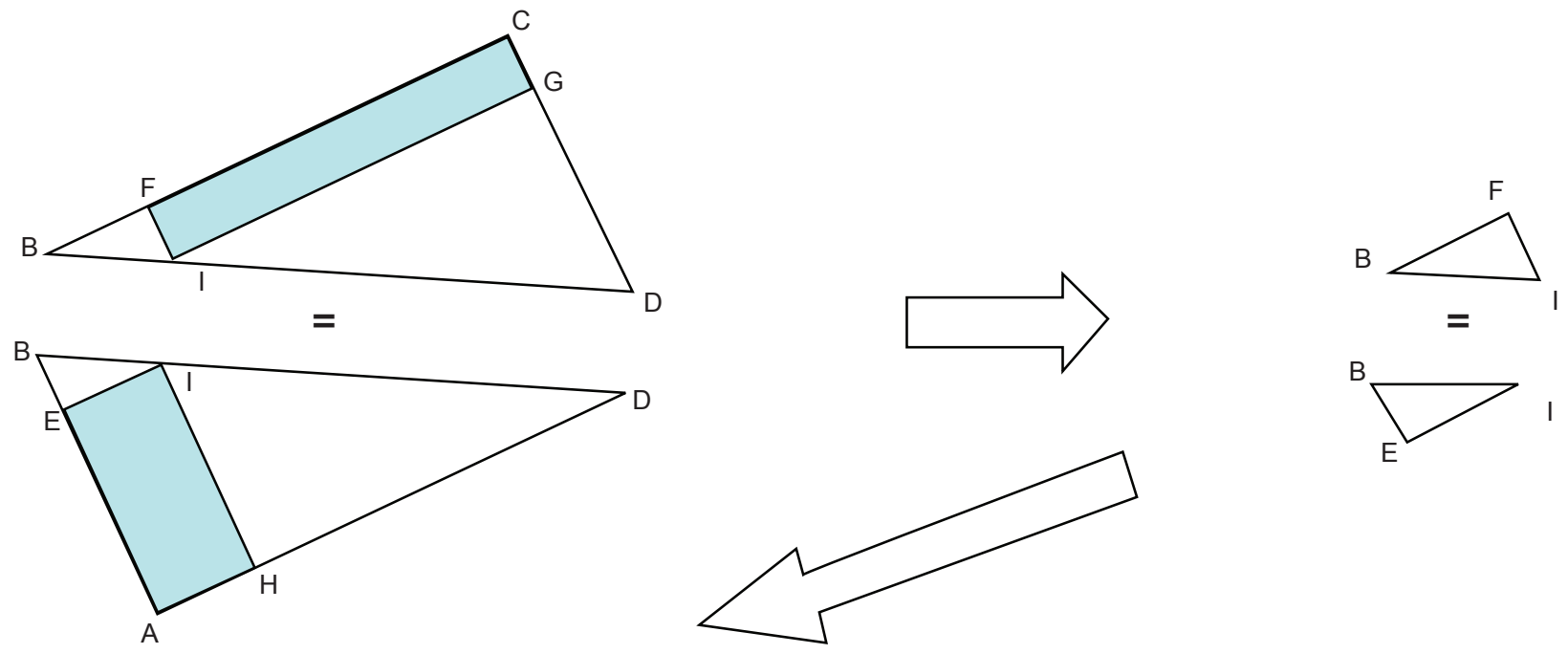

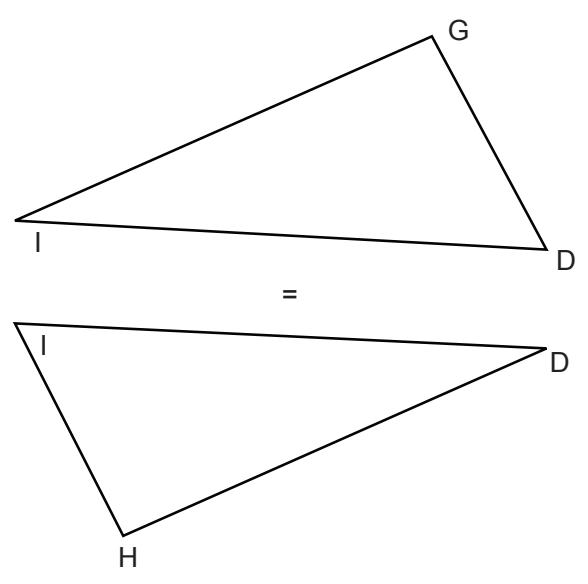
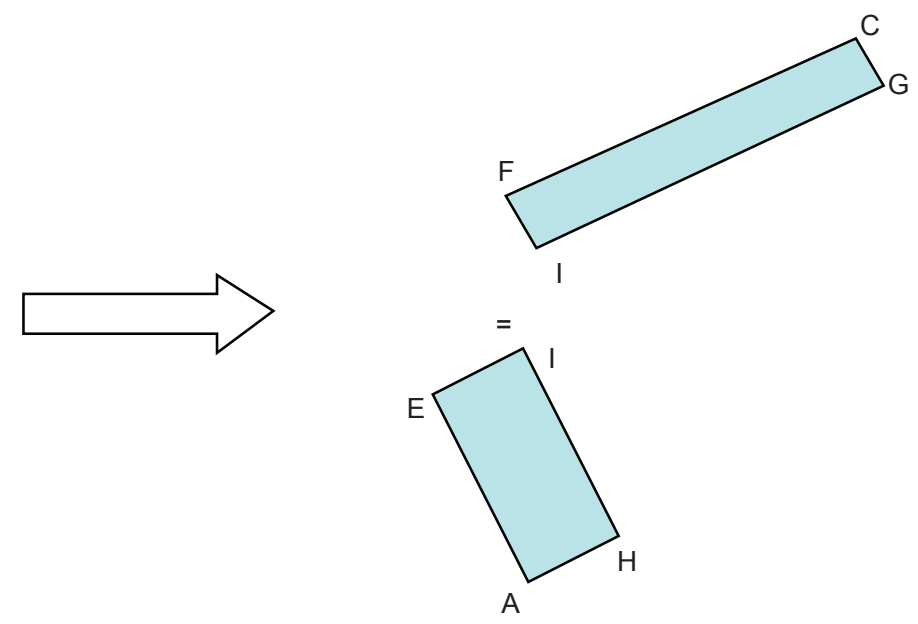

¿Qué justifica esta conjetura?

Mediante una "exploración visual", el estudiantado podría observar que $\triangle A B D \cong \triangle D B C \mathrm{y}$ $\alpha(\triangle A B D)=\alpha(\triangle D B C)$ (Figura 3). Este resultado se puede aprovechar para dos cosas:

- $\quad$ para que la estudiante y el estudiante conjeture que en un rectángulo, la diagonal divide a este en dos triángulos congruentes y, por lo tanto, con la misma área;

- $\quad$ para que, al investigar por qué dicha propiedad se cumple, compruebe que existen dos resultados que apoyan esta conclusión:

$\checkmark \quad$ la diagonal de un paralelogramo divide a este en dos triángulos congruentes;

$\checkmark \quad$ si dos triángulos son congruentes entonces poseen igual área.
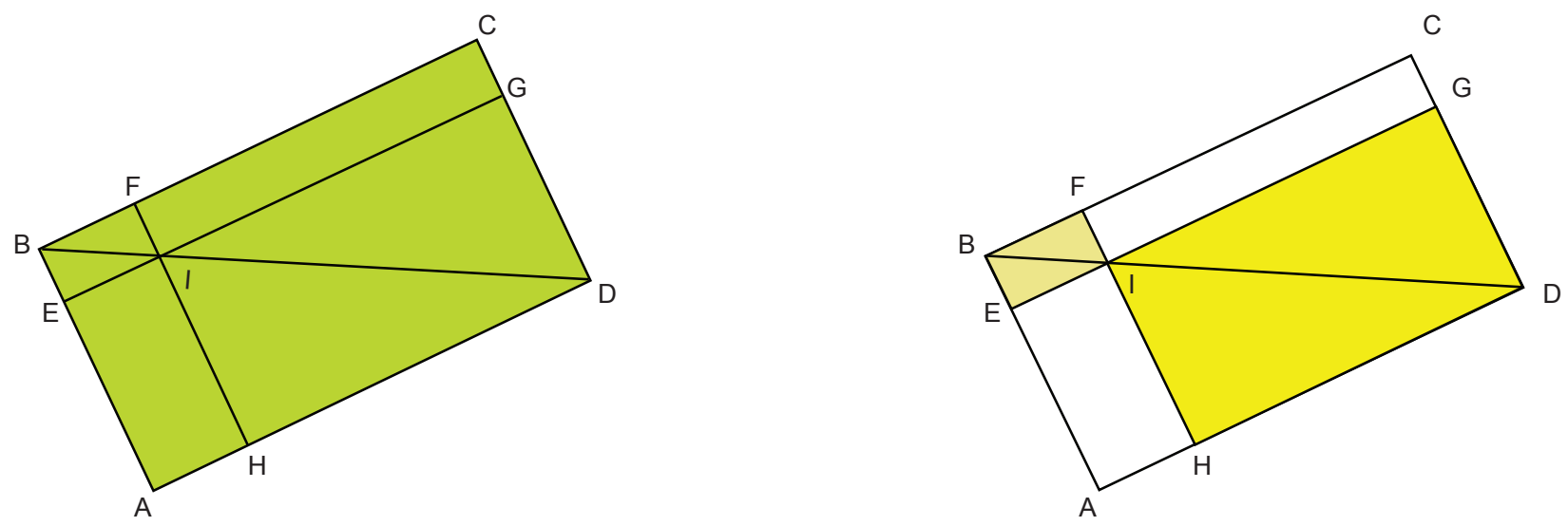

Figura 3. La diagonal de un rectángulo divide a este es dos triángulos congruentes. 
Dado que los cuadriláteros $\square B E I F$ y $\square I H D G$ y son rectángulos con diagonales $\overline{B I}$ y $\overline{D I}$ y (Figura 3), respectivamente, la propiedad anterior permite deducir que:

- $\triangle B E I \cong \triangle I F B$ y $\alpha(\triangle B E I)=\alpha(\triangle I F B)$

- $\triangle I H D \cong \triangle D G I$ y $\alpha(\triangle I H D)=\alpha(\triangle D G I)$

Luego,

- $\alpha(\triangle A B D)=\alpha(\triangle B E I)+\alpha(\Delta I H D)+\alpha(\square A E I H)$

- $\quad \alpha(\triangle D B C)=\alpha(\triangle I F B)+\alpha(\triangle D G I)+\alpha(\square I F C G)$

Así,

$$
\begin{aligned}
& \alpha(\triangle A B D)=\alpha(\triangle D B C) \\
& \Rightarrow \alpha(\triangle B E I)+\alpha(\triangle I H D)+\alpha(\square A E I H)=\alpha(\triangle I F B)+\alpha(\triangle D G I)+\alpha(\square I F C G) \\
& \Rightarrow \alpha(\square A E I H)=\alpha(\square I F C G)
\end{aligned}
$$

El ejemplo anterior permite describir un proceso que no se acaba al "comprobar" visualmente una determinada conjetura, sino que esta se convierte en un punto de partida para que el estudiantado descubra, ofrezca argumentos y elabore razonamientos lógico-matemáticos que la apoyen o la verifiquen.

Para avanzar en el aprendizaje de la geometría las estudiantes y los estudiantes deben pasar de un discurso informal basado en una argumentación descriptiva, a un discurso formal, el cual, apoyado en la visualización, genere un razonamiento que no se basa en una simple descripción de una figura, sino que encadena proposiciones usando inferencia lógica, donde se enuncian definiciones y teoremas (Castiblanco et al., 2004).

Así, si se pretende hacer un cambio en la enseñanza y aprendizaje de la geometría, esta debe centrarse en facilitar los procesos de visualización y justificación, que le permitan el estudiantado "construir su propio conocimiento" y valorar esta disciplina no como un "producto" ya acabado, sino como un campo de descubrimiento y una herramienta para comprender el mundo circundante. La responsabilidad de este cambio recae, en parte, en el personal docente, pues es este grupo quien debe valorar sus prácticas pedagógicas y proponer situaciones de aprendizaje innovadoras y orientadas a incentivar dichos procesos, con el fin de lograr un aprendizaje significativo.

\section{Metodología}

Teniendo como base los referentes teóricos expuestos anteriormente y con el objetivo de lograr una visión general de la situación actual de la enseñanza y aprendizaje de la geometría en la educación secundaria costarricense, se aplicó un cuestionario dirigido a estudiantes, con el propósito de conocer su opinión y elaborar un diagnóstico de lo que sucede en la aulas costarricenses, respecto de la enseñanza de la disciplina.

El cuestionario elaborado y aplicado a las estudiantes y los estudiantes constó de 24 preguntas, las cuales estaban relacionadas con información general, su opinión respecto a qué es aprender geometría, temas en los cuales ha tenido mayor dificultad, tipo de dificultades que ha presentado al 
estudiar geometría, estrategias metodológicas empleadas por la profesora o profesor, estrategias de estudio y uso de la tecnología.

Este cuestionario se elaboró y se validó en el primer semestre 2008. Después de su elaboración el cuestionario se sometió al juicio de expertos para su respetiva revisión; posteriormente fue aplicado a 15 estudiantes de secundaria, que no formaron parte de la población del estudio, con el propósito de analizar sus dudas y comentarios sobre el instrumento y, de ser posible, ser incorporadas en este para su posterior uso. La aplicación se realizó en el segundo semestre del año 2008 a un total de 233 estudiantes de tres instituciones educativas de secundaria (ver Cuadro 1).

Cuadro 1

Distribución de estudiantes de acuerdo al género por institución educativa

\begin{tabular}{|c|c|c|c|c|c|}
\hline \multirow{2}{*}{\multicolumn{2}{|c|}{ Institución }} & \multicolumn{3}{|c|}{ Género } & \multirow{2}{*}{ Total } \\
\hline & & Masculino & Femenino & No respondió & \\
\hline Colegio Técnico Profesional de Puriscal & & 20 & 17 & 1 & 38 \\
\hline Liceo de Santa Gertrudis & & 40 & 43 & 0 & 83 \\
\hline Liceo León Cortés Castro & & 34 & 45 & 1 & 80 \\
\hline No respondió el nombre de la institución & & 17 & 15 & 0 & 32 \\
\hline & Total & 111 & 120 & 2 & 233 \\
\hline
\end{tabular}

La selección de los estudiantes de las tres instituciones educativas se realizó teniendo presente la localización geográfica del colegio y la disposición del profesorado respectivo para la aplicación del cuestionario.

Del total de estudiantes encuestados había 111 hombres y 120 mujeres, distribuidos por nivel de la siguiente manera: 40 sétimo, 78 octavo, 51 noveno, 40 décimo, 23 undécimo y 1 duodécimo (ver Cuadro 2).

Cuadro 2

Distribución de estudiantes según nivel por institución educativa

\begin{tabular}{|c|c|c|c|c|c|c|c|}
\hline \multirow{2}{*}{ Institución } & \multicolumn{6}{|c|}{ Nivel } & \multirow{2}{*}{ Total } \\
\hline & Sétimo & Octavo & Noveno & Décimo & Undécimo & Duodécimo & \\
\hline $\begin{array}{l}\text { Colegio Técnico Profesional de } \\
\text { Puriscal, Puriscal }\end{array}$ & 0 & 27 & 10 & 0 & 0 & 1 & 38 \\
\hline Liceo de Santa Gertrudis, Grecia & 13 & 22 & 19 & 18 & 11 & 0 & 83 \\
\hline Liceo León Cortés Castro, Grecia & 16 & 19 & 15 & 21 & 9 & 0 & 80 \\
\hline $\begin{array}{l}\text { No respondió en nombre de la } \\
\text { institución }\end{array}$ & 11 & 10 & 7 & 1 & 3 & 0 & 32 \\
\hline Total & 40 & 78 & 51 & 40 & 23 & 1 & 233 \\
\hline
\end{tabular}


A continuación se presentan los resultados más relevantes.

\section{Análisis de resultados}

Al grupo de estudiantes encuestados se le pidió completar la frase "Para mí aprender geometría es...” Aunque se obtuvieron variadas respuestas, la siguiente categorización resume los comentarios realizados por los estudiantes.

Tabla 3

Comentarios realizados por el estudiantado para completar la frase "Para mí aprender geometría es..."

\begin{tabular}{lcc}
\hline \multicolumn{1}{c}{ Frase } & Absoluto & Porcentaje \\
\hline Algo importante, interesante & 72 & 30,9 \\
Difícil, una situación que no es de mi agrado, aburrido & 70 & 30,0 \\
Estudiar propiedades de las figuras & 19 & 8,2 \\
Fácil & 18 & 7,7 \\
Una forma de aprender, pensar y razonar & 11 & 4,7 \\
Aprender matemática & 9 & 3,9 \\
Poco útil, una pérdida de tiempo & 4 & 1,7 \\
Otros & 14 & 6,0 \\
No respondió & 16 & 6,9 \\
\hline
\end{tabular}

Como lo muestra el Cuadro \# 3, aunque un importante número de estudiantes señala que estudiar geometría es algo importante e interesante, una cifra no despreciable de alumnas y alumnos señala que es difícil y aburrido. Entre algunas de las respuestas dadas por están: es una materia muy fácil, pero a veces es muy aburrida; la geometría es una forma de aprender e interesante, pero al mismo tiempo cansada y difícil; estudiar geometría es aprender a hacer muchas cosas matemáticas; es como aprender cualquier otra cosa, da igual; es muy importante porque la geometría es parte de los que nos rodea; es difícil por las fórmulas y aburrido; es algo muy interesante y divertido por que nos entretenemos haciendo los ejercicios, cada vez vamos aprendiendo más; es algo importante, pero un poquito enredado; la geometría es muy importante, ya que se puede utilizar tanto en la vida cotidiana como en el colegio y la universidad; bonito, pero todo depende de la profesora o profesor; es fácil pero no me va a servir de nada; depende de la forma en que la profesora o el profesor la enseñe es fácil o difícil; la geometría es lo más aburrido del mundo; es una materia muy pero muy difícil y complicada, además de aburrida; es difícil, pero con dedicación se logra lo mejor en matemáticas; es simplemente solo para pasar el colegio; aprender geometría es saber fórmulas y aplicar conocimientos con los triángulos para poder avanzar en el mundo de las matemáticas.

Se puede observar que el estudio de esta disciplina se asocia, por varias y varios estudiantes, como aprender propiedades de las figuras geométricas, mientras que otras y otros consideran que estudiar geometría no tiene ninguna importancia y particularidad en especial, simplemente es parte de las matemáticas. Es importante señalar que el estudiantado indica que la memorización 
de fórmulas y propiedades de las figuras geométricas y el factor "profesora o profesor" son componentes que influyen en la enseñanza y aprendizaje de la geometría.

Respecto de la importancia de aprender geometría en secundaria, 182 estudiantes respondieron afirmativamente y 46 indicaron que el estudio de esta disciplina no tiene relevancia en el colegio. Entre las razones que apuntaron para justificar que el estudio de esta disciplina no es importante están:

- $\quad$ Porque es muy aburrido.

- $\quad$ Porque no veo el modo de utilizarla ni ahora ni nunca y pienso que mucha gente también lo piensa así.

- $\quad$ Porque algunas veces no se necesita en el futuro.

- $\quad$ Porque no tiene nada que ver con la carrera que yo quiero estudiar.

- $\quad$ Porque es muy difícil y sirve de muy poco, depende de la carrera que se escoja.

- $\quad$ No me gusta, es muy aburrido, innecesario, es el tema que complica la resolución del examen de matemática en bachillerato

- $\quad$ Porque creo que es un tema fácil, por lo cual en la secundaria no sería tan importante.

- $\quad$ Porque eso se ve en la escuela y no precisa volver a verlo.

El principal argumento que brindan para justificar la poca importancia que se le da al estudio de la geometría, es que no le encuentran una utilidad a los contenidos que se desarrollan en secundaria y consideran que lo que aprendieron en la escuela es suficiente. Razones opuestas dan quienes consideran que aprender geometría en secundaria es importante, pues estos indican que los contenidos que se desarrollan son aplicables en la vida cotidiana. Pareciera, entonces, según las razones dadas por las estudiantes y los estudiantes, que la importancia de aprender la disciplina está asociada a la aplicabilidad de esta, es decir, sería importante aprender solamente lo que se puede utilizar en la vida.

Respecto de los temas de geometría en los cuales el estudiantado ha tenido mayor dificultad, en orden descendente, se encuentran: ángulos entre dos rectas paralelas y una transversal, semejanza de triángulos y teorema de Thales, rectas notables en un triángulo (altura, mediana, mediatriz, bisectriz) y triángulos (clasificación, desigualdad triangular, ángulo externo, entre otros).

Al realizar el análisis por nivel, los temas en los cuales señalaron haber tenido mayor dificultad son: rectas notables en un triángulo (altura, mediana, mediatriz, bisectriz) (sétimo año), semejanza de triángulos y teorema de Thales (octavo año), teorema de Pitágoras (noveno año), ángulos entre dos rectas paralelas y una transversal (décimo y undécimo año), áreas sombreadas y áreas y volúmenes de sólidos (undécimo año). Es importante señalar que el 71,2\% de estudiantes señala que su profesora o profesor de Matemáticas no le da una importancia específica a algún tema de geometría.

Respecto a las dificultades que las estudiantes y los estudiantes presentan al estudiar geometría se encuentran: resolver un problema algebraicamente; calcular perímetros, áreas y volúmenes, debido a que no identifican cuál fórmula aplicar y dificultad para interpretar qué es lo que dice un problema. Al realizar el análisis por nivel, se puede observar que en el ciclo diversificado (décimo y undécimo año) la principal dificultad que presentan es interpretar lo que dice un problema. La principal dificultad de las alumnas y alumnos de sétimo, octavo y noveno año, es, respectivamente, comprender las fórmulas del perímetro, áreas y volúmenes y aprender las definiciones; resolver una situación problema algebraicamente y dificultad para extraer información de un dibujo geométrico. 
Nuevamente se pone de manifiesto que la memorización constituye, para el estudiantado, un importante elemento en el aprendizaje de la geometría, dada la "necesidad" de saber fórmulas, definiciones y características.

Si se analizan, de acuerdo con la opinión del grupo estudiantil, las actividades, estrategias, técnicas o métodos que las profesoras y profesores usualmente realizan durante el desarrollo de sus clases, se puede observar que la mayoría del estudiantado indica que el profesorado desarrolla su clase de manera "tradicional", es decir, inicia definiendo los conceptos, esto es, con la teoría, y luego realiza algún ejemplo en la pizarra. Posteriormente deja práctica. Actividades como juegos para enseñar algunos temas, uso de la tecnología, uso de material concreto y lecturas complementarias son actividades que, según el criterio de estudiantes, son poco utilizadas.

Debido a este corte tradicional de la enseñanza de la geometría, actividades como realizar dibujos que representen situaciones geométricas con el fin de comprender la materia estudiada, visualizar y conjeturar son poco utilizadas.

Entre las principales "estrategias" empleadas por los estudiantes para estudiar geometría están: leer definiciones y entender los ejercicios que la profesora y el profesor hace en clase; aprender de memoria las definiciones y fórmulas para calcular perímetros, áreas y volúmenes; tratar de identificar cuál es el procedimiento algebraico que permite resolver un problema, para aplicarlo a otros similares; aprender "trucos" en la calculadora que les ayuden en el momento del examen y realizar listas de ejercicios para desarrollar la habilidad práctica.

Respecto de los recursos que las profesoras y profesores utilizan para desarrollar la clase de geometría, los estudiantes señalan que hay poca diversidad. Entre los principales recursos que utiliza el profesorado para desarrollar la clase de geometría son, en orden de importancia y según la opinión estudiantes, la pizarra, la tiza o pilot y borrador, material fotocopiado y libro de texto. Estos recursos y su importancia son consecuentes cuando se realiza el análisis por nivel.

Según la opinión de las estudiantes y los estudiantes, el uso de cartulina, periódico, tijeras, goma u otro material concreto, algún programa computacional especial para geometría y equipo tecnológico (computadora, proyector de multimedia, calculadoras, entre otros) son poco empleados por las profesoras y profesores.

Al grupo de estudiantes también se les interrogó sobre las habilidades, hábitos o actitudes que ellos consideran que se deben poseer para tener éxito en geometría. En forma general, consideran que para tener éxito en geometría las tres principales habilidades, hábitos o actitudes que se deber tener son:

- Saber utilizar la calculadora para realizar cálculos.

- Capacidad para memorizar definiciones, fórmulas y teoremas.

- Capacidad para entender los dibujos geométricos.

Sin embargo, al realizar el análisis por nivel se puede observar que para el grupo estudiantil de los niveles de sétimo y octavo año lo principal es tener capacidad para memorizar definiciones, fórmulas y teoremas; para los de noveno año, capacidad para entender los dibujos geométricos; para los de décimo, saber utilizar la calculadora para realizar cálculos y para los de undémico año, realizar listas de ejercicios para desarrollar la habilidad práctica.

Cuando a las estudiantes y los estudiantes se les preguntó si utilizaban algún recurso tecnológico para estudiar geometría, de los 233 estudiantes que contestaron, $122(52,4 \%)$ señaló que sí, $104(44,6 \%)$ indicó que no y $7(3 \%)$ no contestó, y resultó la calculadora como el recurso más utilizado. Solamente 35 estudiantes (15\%) señaló que utilizaba la computadora. Un porcentaje muy 
bajo $(13,7 \%)$ indica que ha utilizado Internet para documentarse sobre temas relacionados con la geometría.

Un comportamiento similar se presenta cuando se les pregunta si su profesora o profesor ha utilizado algún equipo tecnológico para enseñar geometría, pues sólo 64 estudiantes $(27,5 \%)$ señaló afirmativamente e indicó que la calculadora científica era el recurso más utilizado.

Respecto a si les gustaría recibir clases de geometría en un laboratorio de cómputo, donde ellos pudiesen utilizar una computadora, $75,5 \%$ contestó que sí y $24 \%$ señaló que no. Los estudiantes señalan que con el uso de la computadora el estudio de la geometría se haría más fácil, se podría trabajar una mayor cantidad de ejemplos, le tomarían más importancia y aprenderían más sobre el tema. Además, señalan que les permitiría hacer más práctica que en un libro, jugar con la geometría y podrían hacer figuras geométricas tridimensionales, sería menos aburrido, aprenderían con memoria visual, se cambiaría la rutina y se podría realizar trabajos más buenos, más ordenados y permitiría aclarar algunas dudas.

Por otra parte, algunas y algunos estudiantes señalan que el uso de la computadora no se podría implementar en las clases de geometría porque "la práctica hace al maestro" y las computadoras dan la respuesta, lo que provoca pérdida del trabajo "realizado a mano"; además, sería mucho más difícil y nadie pondría atención ni dejarían poner atención, ya que la atención se centraría en las computadoras y no en el tema que se está desarrollando.

En relación con este tema, los estudiantes señalan posiciones diversas. Sin embargo, la profesora y el profesor deben tener presente que no todas ni todos los estudiantes aprenden de la misma forma y que algunas y algunos estudiantes podrían presentar resistencia al uso de la tecnología. Cómo se use y de qué forma es responsabilidad única de la docente y el docente.

Los resultados presentados anteriormente dan una visión de lo que ocurre en las lecciones de geometría. Corresponde a la concepción de las estudiantes y los estudiantes. Sin embargo, permite identificar algunos puntos a los cuales se deben prestar especial atención y que serán comentados en la sección posterior.

El propósito no es buscar culpables respecto al porqué o no de determinada situación, sino poder plantear acciones integrales que contribuyan a mejorar el proceso de enseñanza y aprendizaje de la geometría.

\section{Conclusiones}

Los resultados señalan que las clases de geometría se han basado en un sistema tradicional de enseñanza, donde es la profesora o el profesor el principal actor de este proceso. Ellos presentan la teoría, desarrollan ejemplos y aportan los ejercicios que deben ser resueltos por las estudiantes y los estudiantes. Sin embargo, estos ejercicios enfatizan en la aplicación de fórmulas y aspectos memorísticos donde se deja de lado procesos de visualización, argumentación y justificación, y se incentiva la búsqueda del "procedimiento algoritmo o algebraico" más adecuado para dar solución a las actividades que se proponen.

Debido a ello, la geometría se presenta al alumnado como una "receta" de definiciones, fórmulas y teoremas totalmente alejada de su realidad y donde los ejemplos y ejercicios no poseen ninguna relación con su contexto. Esta situación provoca que el estudiantado no considere importante el estudio de esta disciplina porque no es aplicable a la vida cotidiana.

Aunque no existe un tema especial al cual el cuerpo docente le otorge alguna importancia especial, las estudiantes y los estudiantes reconocen que los temas en los que han tenido mayor 
dificultad son: ángulos entre dos rectas paralelas y una transversal, semejanza de triángulos y teorema de Thales, rectas notables en un triángulo (altura, mediana, mediatriz, bisectriz) y triángulos (clasificación, desigualdad triangular, ángulo externo, entre otros). En general, señalan que las principales dificultades que poseen al estudiar geometría son:

- $\quad$ resolver un problema algebraicamente;

- calcular perímetros, áreas y volúmenes, debido a que no identifican cuál fórmula aplicar;

- $\quad$ dificultad para interpretar qué es lo que dice un problema.

Si bien todos los temas son importantes y la profesora y el profesor debe enfatizar en cada uno de ellos, conocer los contenidos en los cuales se presenta mayor dificultad y el tipo de problema, le implica a la docente y al docente otorgar una especial importancia en ellos y emplear estrategias metodológicas que faciliten el aprendizaje.

El uso de recursos para la enseñanza de la geometría es una responsabilidad docente. Seleccionar los más adecuados, dependen de ellos. Aunque la tecnología es uno de estos, la utilizan muy poco. El recurso más utilizado es la calculadora. Sin embargo, otros como juegos, uso de material concreto, algún programa computacional especial para geometría y lecturas complementarias son poco utilizadas.

Esto se debe, principalmente, a la forma tradicional de enseñanza en la que se desarrolla la disciplina en las aulas de secundaria, donde los principales recursos utilizados son pizarra, tiza o pilot y borrador, material fotocopiado y libro de texto.

Esta concepción de la enseñanza de la geometría desarrolla por el estudiantado a partir de su experiencia en las aulas, puede ser la causa que lo ha llevado a pensar que para lograr éxito en geometría hay que saber utilizar la calculadora para realizar cálculos, tener capacidad para memorizar definiciones, fórmulas y teoremas, poseer capacidad para entender los dibujos geométricos y realizar listas de ejercicios para desarrollar la habilidad práctica.

Los resultados obtenidos para nuestro contexto coinciden con los señalados por Goncalves (2006), quien señala que las lecciones de geometría se han desarrollado de manera abstracta, sin proporcionarles a los estudiantes ejemplos reales que les faciliten un mejor entendimiento de los contenidos y donde estos tienen que recurrir a memorizar las demostraciones de los teoremas o las formas de resolver los problemas, pues es la única manera de aprobar los exámenes.

Si se comparan nuestros resultados con los expuestos por Barrantes y Blanco $(2005,2004)$, muchas de las concepciones del estudiantado español coinciden con las expuestas en este documento. Aunque ese no es el objetivo de la investigación, la problemática en la enseñanza de la geometría plantea un desafío a todos los involucrados en el proceso de enseñanza y aprendizaje de las Matemáticas para hallar alternativas de solución, pues la enseñanza de la geometría se ha desvirtuado y se han dejado de lado procesos de razonamiento, argumentación y visualización, los cuales son trascendentales para el aprendizaje.

La enseñanza de la geometría debe centrarse en desarrollar, en el estudiantado, habilidades para la exploración, visualización, argumentación y justificación, donde más que memorizar puedan descubrir, aplicar y obtener conclusiones. El cuerpo docente debe interiorizar que en este proceso no es él el principal actor, sino los estudiantes, los cuales deben ser promotores de su aprendizaje a partir de su "guía", donde las actividades planteadas y los recursos disponibles faciliten y contribuyan en dicho proceso. 


\section{Referencias bibliográficas}

Abrate, R.; Delgado, G. y Pochulu, M. (2006). Caracterización de las actividades de Geometría que proponen los textos de Matemática. Revista Iberoamericana de Educación, 39(1), 1-9. Recuperado el 22 de octubre de 2007 en http://www.rieoei.org/deloslectores/1290Abrate.pdf

Almeida, M. (2002). Desarrollo Profesional Docente en Geometría: análisis de un proceso de Formación a Distancia. (Memoria de tesis doctoral). Departamento de Didáctica de las Ciencias Experimentales y de las Matemáticas. Universidad de Barcelona, Barcelona. Recuperado el 22 de octubre de 2007 en http://www.tesisenxarxa.net/TESIS UB/AVAILABLE/TDX-1008102120710//TOL119.pdf

Báez, R. \& Iglesias, M. (2007). Principios didácticos a seguir en el proceso de enseñanza y aprendizaje de la geometría en la UPEL “El Mácaro”. Enseñanza de la Matemática, Vols. 12 al 16, Número extraordinario, 67-87.

Barrantes, M. (2004). Recuerdos, expectativas y concepciones de los estudiantes para Maestro sobre la geometría escolar y su enseñanza-aprendizaje. (Tesis doctoral). Departamento de Didáctica de las Ciencias Experimentales y de las Matemáticas, Facultad de Educación, Universidad de Extremadura. España.

Barrantes, M. y Blanco, L. J. (2004). Recuerdos, expectativas y concepciones de los estudiantes para Maestro sobre la geometría Escolar. Enseñanza de las Ciencias 22(2), 241-250.

Barrantes, M. y Blanco, L. J. (2005). Análisis de las concepciones de los profesores en formación sobre la enseñanza y aprendizaje de la geometría. Números, 62, 33-44.

Castiblanco, A., Urquina, H., Camargo, L. y Acosta, M. (2004). Pensamiento Geométrico y Tecnologías Computacionales. Colombia: Ministerio de Educación Nacional. Enlace Editores Ltda.

Costa Rica. Ministerio de Educación Pública. (2005). Programas de estudio de Matemática. III Ciclo. San José, Costa Rica.

Gamboa, R. y Morales, Y. (2008). Alcances y precauciones sobre el uso de software de geometría dinámica tipo constructivo-deductivo. Memoria del X Evento Internacional MATECOMPU [Memoria digital en CD]. Matanzas, Cuba.

Goncalves, R. (2006, Enero-Junio). ¿Por qué los estudiantes no logran un nivel de razonamiento en la geometría? Revista Ciencias de la Educación, 1(27), 83-98.

Hernández, V. \& Villalba, M. (2001). Perspectivas en la Enseñanza de la geometría para el siglo XXI. Documento de discusión para estudio ICMI. PMME-UNISON. Traducción del documento original. Recuperado el 18 de octubre de 2007 en http://www.euclides.org/menu/ articles/article2.htm 
Jones, K. (2002). Issues in the Teaching and Learning of Geometry. En L. Haggarty (Ed.), Aspects of Teaching Secondary Mathematics. Perspectives on practice (pp. 121-139). London: RoutledgeFalmer.

National Council of Teachers of Mathematics [NCTM]. (2000). Principles and standards for school mathematics. Reston, VA: National Council of Teachers of Mathematics.

Torregosa, G. \& Quesada, H. (2007, Julio). Coordinación de procesos cognitivos en Geometría. Revista Latinoamericana de Investigación en Matemática Educativa, 10(2), 275-300. 